J. Clin. Chem. Clin. Biochem.

Vol. 24, 1986, pp. 179-184

(C) 1986 by Walter de Gruyter \& Co. Berlin - New York

\title{
Caeruloplasmin in der Diagnostik gynäkologischer Tumoren
}

\author{
Von Th. Schultek, B. Tode und W. G. Wood \\ Klinik für Innere Medizin (Direktor: Prof. Dr. P. C. Scriba) Medizinische Universität zu Lübeck
}

(Eingegangen am 30. Juli/27. November 1985)

Zusammenfassung: Es wurde die Caeruloplasmin-Konzentration im Serum von Patientinnen mit Ovarial-, Collum- und Corpuskarzinomen untersucht. Die Konzentration im Serum wurde aufgrund seiner enzymatischen Aktivität und mittels der chemiluminometrischen Verfahren ILMA (immunoluminometrischer Assay) und SPALT (solid phase antigen luminescence technique) bestimmt. Der Median der mit dem SPALT-Assay gemessenen Caeruloplasmin-Konzentration der Tumor-Patientinnen (Median: 0,78 g/1) war signifikant höher als der nach den beiden anderen Verfahren bestimmte (enzymatische Meßmethode: Median 0,26 g/l, ILMAAssay: Median 0,44 g/l). Im Vergleich mit dem Referenzkollektiv (Median 0,29 g/l) zeigte sich eine deutlich erhöhte mittlere Konzentration bei Tumorpatientinnen, deren Caeruloplasmin-Gehalt mit dem SPALT-Assay gemessen wurde. Die nach den beiden anderen Methoden gemessenen Konzentrationen unterschieden sich nicht vom gesunden Referenzkollektiv. Mit dem SPALT-Assay werden im Gegensatz zu den beiden anderen Verfahren möglicherweise paraneoplastische Substanzen mit Caeruloplasmin-ähnlicher Immunreaktivität erfaßt.

Die Medianwerte der tisșue polypeptide antigen (TPA)-Konzentration der Tumorpatientinnen waren gegenüber dem gesunden Vergleichskollektiv signifikant erhöht, der Median der Konzentration von carcinoembryonalem Antigen (CEA) entsprach dem Referenzkollektiv.

\section{The use of caeruloplasmin in the diagnosis of gynaecological tumours}

Summary: Serum caeruloplasmin levels were measured in women with ovarian, collum and corpus carcinomata using three different assay methods. The enzyme assay gave a median value of $0.26 \mathrm{~g} / \mathrm{l}$, the SPALT (solid phase antigen luminescence technique) a median value of $0.78 \mathrm{~g} / 1$ and the ILMA (immunoluminometric assay) a median of $0.44 \mathrm{~g} / 1$. A group of blood donors, taken as the reference group, had a median value of $0.29 \mathrm{~g} / 1$ in the SPALT assay, which was significantly lower than in the tumour group measured by this method. The ILMA and enżymatic assay values were not significantly different from those measured in the reference group.

The differences in the SPALT method can be ascribed to the possibility that this assay measures not only "intact" caeruloplasmin but also paraheoplastic substances with "caeruloplasmin-like immunoactivity".

The median of the concentration of tissue polypeptide antigen (TPA) was significantly different from those measured in the reference group. The concentrations of carcinoembryonic antigen (CEA) were not different from the reference group.

\section{Einfuihrung}

Caeruloplasmin ist ein Protein der $\alpha_{2}$-Globulinfraktion des Serums, bestehend aus zwei leichten und zwei schweren Polypeptidketten. Das Molgewicht der
$\alpha$-Kette (N-terminal: Valin) beträgt 16000 , das der $\beta$ Kette 59000 (N-terminal: Lysin) (7). Die Eigenschaft des Caeruloplasmins als kupferbindendes Protein wurde schon vor längerer Zeit beschrieben. Andere 
in vivo-Funktionen dieser Substanz sind, außer einer Oxidase-Aktivität, noch nicht bekann̈t. Von besonderem klinischen Interesse ist, $\mathrm{da} \beta$ die Konzentration von Caeruloplasmin im Serum bei Patienten mit Wilson'scher Erkrankung signifikant erniedrigt ist. Bei Tumorerkrankungen kommt es dagegen oft, parallel zu einem Anstieg des Kupfers im Serum, zu einer Erhöhung der Caeruloplasmin-Konzentration.

In dieser Studie untersuchten wir die CaeruloplasminKonzentrationen im Serum von Patientinnen mit gynäkologischen Tumoren. Die Bestimmung erfolgte unter Anwendung dreier unterschiedlicher Untersuchungsmethoden: enzymatisch und immunologisch mittels zweier von uns entwickelter luminometrischer Assays. Die Ergebnisse der nach den verschiedenen Methoden erhaltenen Konzentrationen werden untereinander und mit den konventionellen Tumormarkern carinoembryonales Antigen (CEA) und tissue polypeptide antigene (TPA) verglichen.

\section{Material und Methoden}

\section{Probanden}

Untersucht wurden die Seren von $n=64$ Patientinnen mit Ovarial-, Collum- und Corpuskarzinomen der Tumorstadien III und IV nach FIGO ${ }^{1}$ ), ${ }^{2}$ ). Die Seren waren bis zur Untersuchung bei $-20^{\circ} \mathrm{C}$ tiefgefroren, allerdings nie länger als 14 Tage. Als Vergleichskollektiv dienten 74 gesunde Blutspender unterschiedlichen Geschlechts mit normalen Aminotransferasen, $\gamma$-Glutamyltransferasen und einer Blutsenkungsgeschwindigkeit unter $30 \mathrm{~mm}$ nach Westergren. Ausgeschlossen wurden Raucher. Sämtliche Referenzwerte lagen innerhalb der 2,5 und 97,5 Percentile (Tab. 1).

1) Wir danken der Klinik für Gynäkologie und Geburtshilfe der Medizinischen Hochschule Lübeck (Direktor: Prof. Dr. $F$. Oberheuser) für die Überlassung des Untersuchungsmaterials.

2) FIGO: Federation international de gynaecologie et d'obstetrie.

Tab. 1. Caeruloplasmin - CEA - TPA

Referenzwerte eines gesunden Kollektivs (Blutspender beiderlei Geschlechts).

\begin{tabular}{|c|c|c|c|c|}
\hline & $\begin{array}{l}\text { Caerulo } \\
\text { SPALT } \\
(\mathrm{g} / \mathrm{l})\end{array}$ & $\begin{array}{l}\text { plasmin } \\
\text { ILMA } \\
(\mathrm{g} / \mathrm{l})\end{array}$ & $\begin{array}{l}\text { TPA } \\
(U / 1)\end{array}$ & $\begin{array}{l}\text { CEA } \\
(\mu \mathrm{g} / \mathrm{l})\end{array}$ \\
\hline Anzahl & 74 & 50 & 108 & 108 \\
\hline Mittelwert & 0,34 & 0,30 & 36,7 & 1,32 \\
\hline $\begin{array}{l}\text { Percentile } \\
2,5 \\
16 \\
50 \text { (Median) } \\
84 \\
97,5\end{array}$ & $\begin{array}{l}0,06 \\
0,18 \\
0,29 \\
0,50 \\
0,70\end{array}$ & $\begin{array}{l}0,10 \\
0,18 \\
0,27 \\
0,43 \\
0,59\end{array}$ & $\begin{array}{l}17 \\
27 \\
36 \\
48 \\
58\end{array}$ & $\begin{array}{l}0,25 \\
0,66 \\
1,33 \\
1,85 \\
2,29\end{array}$ \\
\hline Mittelwert/Median & 1,17 & 1,13 & 1,02 & 0,99 \\
\hline
\end{tabular}

\section{Caeruloplasmin}

Die Bestimmung der Caeruloplasmin-Konzentration im Serum wurde nach folgenden Verfahren durchgeführt:

\section{Methode A}

Messung der enzymatischen Oxidation von $p$-Phenylendiamin durch Caeruloplasmin unter Verwendung der 2-Punkt-Methode (3). Bei der Berechnung der Konzentrationen verwendeten wir nicht den bekannten Umrechnungsfaktor von Enzymaktivität zu Konzentration, sondern erstellten eine Standardkurve.

\section{Methode B}

Immunoluminometrische Bestimmung nach der Festphasenantigen-Lumineszenztechnik (SPALT) (11). Hierzu wurden $100 \mu \mathrm{l}$ einer 1:50 verdünnten Serumprobe (entsprechend verdünnte Standards von 2,5 bis $0,08 \mathrm{~g} / \mathrm{l}$; Caeruloplasmin, Sigma Nr. C4770) mit $100 \mu \mathrm{l} 1: 1000$ verdünntem Sheep-anti-human Caeruloplasmin-Antikörper (Seward Laboratories, London) $10 \mathrm{Mi}$ nuten inkubiert. Anschließend erfolgte eine Inkubation mit einer Antigen-beschichteten Polystyrolkugel für 55 Minuten bei Raumtemperatur. Nach Waschen mit 4L-PBS-Tween (Tab. 2) 55 Minuten Inkubation mit einem Antikörper gegen Schaf-IgG (DAKOPATTS, Hamburg), markiert mit $\mathrm{N}$-6-(4-aminobutylN-ethyl)isoluminol)hemisuccinamid (ABEI-H). Die luminometrische Messung erfolgte in einem vollautomatischen Luminometer der Fa. Bertold, Wildbad, (LB-950 T) in einem 1-Dispenserbetrieb (12). Jeder Küvette wurden $300 \mu$ l eines $\mathrm{NaCl} / \mathrm{Mikro}$ peroxidase-Gemisches als Startreagenz im Volumenverhältnis $2: 1$ vorgegeben.

\section{Methode C}

Immunoluminometrische Analyse mit Antihuman-Caèruloplasmin-Antikörpern beschichteten Polystyrolkugeln (ILMA) (11).

Anti-human-Caeruloplasmin-beschichtete Kugeln (Sheep-antihuman-Caeruloplasmin-Antikörper, gereinigte IgG-Fraktion, Seward Laboratories, London) wurden mit $20 \mu$ l Serum bzw. Standard (jeweils 1:1000 vorverdünnt) in $2000 \mu \mathrm{l}$ 4L-PBSTween eine Stunde bei Raumtemperatur unter Schütteln inkubiert. Nach zweimaligem Waschen eine Stunde Inkubation mit Anti-human-Caeruloplasmin (Rabbit-a-h-Caeruloplasmin, mittels Adsorption und Affinitätsch̆romatographie gereinigte IgG-Fraktion, DAKOPATTS, Hamburg) (1:1000 verdünnt), markiert mit 7-N-(4-aminobutyl)-N-ethyl) naphthalin-1,2-dicarbonsäurehydrazid (ABEN-H) ${ }^{3}$ ). Anschließend luminometrische Messung wie oben beschrieben.

3) Die Substanz wurde uns freundlicherweise von Dr. $H$. Schroeder (Miles Laboratories, Elkhart, Indiana, USA) überlassen.

Tab. 2. „4 L-PBS-Tween“ Pufferlösung.

\section{L-Stammlösung}

$121,14 \mathrm{~g}$ Tris-Puffer

$+800 \mathrm{ml} \mathrm{H}$ O (mit $250 \mathrm{~g} / \mathrm{kg} \mathrm{HCl}$ auf $\mathrm{pH} 7,4$ einstellen)

$+14,9 \mathrm{~g} \mathrm{KCl}$

+20 g Rinder-Serum-Albumin

PBS-Tween-Stammlösung

$1000 \mathrm{ml} \mathrm{0,5} \mathrm{mol} / 1$ Phosphatpuffer $\mathrm{pH} 7,5$

$+90 \mathrm{~g} \mathrm{NaCl}$

$+5 \mathrm{ml}$ Tween

\section{L-PBS-Tween Gebrauchslösung}

$450 \mathrm{ml} \mathrm{H}_{2} \mathrm{O}$

$+25 \mathrm{ml} 4$ L-Stammlösung

$+25 \mathrm{ml}$ PBS-Tween-Stammlösung 
Zur Erstellung der Standardkurven verwandten wir eine Lösung von $50 \mathrm{~g} / \mathrm{l}$ Caeruloplasmin (Sigma, München, D., No. c-4770), die mit Assaypuffer verdünnt wurde (Spannbreite $0-5 \mathrm{~g} / \mathrm{l}$ ).

Eine Verdünnung eines kommerziellen humanen Referenzserums (Protein-Standard-Plasma, Behring-Institut) ergab einen parallelen Verlauf der identischen Konzentration.

Die CEA-Bestimmung der Patientenseren wurde mit dem CEAEnzym-Immunoassay von Abbott (Wiesbaden) und die TPAMessung mit dem Prolifigen-TPA-RIA-Kit von Sangtec (Bromma, S) durchgeführt. Die ermittelten Konzentrationen wurden mit entsprechenden gesunder Blutspender verglichen. Die Referenzbereiche sind in Tabelle 1 aufgeführt.

Mit $2 \mathrm{ml}$ Serum einer Patientin mit Corpuskarzinom Stadium III wurde eine Gelfiltrations-Chromatographie (Säule $=2 \mathrm{~cm}$ $\times 20 \mathrm{~cm}$, Säulenmaterial: Ultrogel AG (LKB, Bromma S), Elutionsmittel: Phosphatpuffer $0,05 \mathrm{~mol} / 1, \mathrm{pH}=7,6$ ) durchgefübrt. In den einzelnen Fraktionen wurde Caeruloplasmin immunoluminometrisch parallel mit dem ILMA und dem SPALT bestimmt. Den SPALT führten wir bei diesem Versuch mit zwei unterschiedlichen Antikörpern durch:

SPALT 1 mit Rabbit-a-h-Caeruloplamin (DAKOPATTS, Hamburg und

SPALT 2 mit Sheep-a-h-Caeruloplasmin (Seward Laboratories, London).

\section{Ergebnisse}

Für die enzymatisch bestimmten CaeruloplasminKonzentrationen wurde eine mittlere Konzentration eines erwachsenen Referenzkollektivs von $0,44 \pm$ $0,14 \mathrm{~g} / 1$ (3) zugrunde gelegt. Die immunbiochemisch nach dem ILMA- und SPALT-Verfahren bestimmten Caeruloplasmin-Konzentrationen des gesunden Referenzkollektivs sind in Tabelle 1 aufgeführt.

Bei den Patientinnen mit Tumor bestimmten wir enzymatisch einen Medianwert von 0,27 g/1. Die immunoluminometrisch gemessene Caeruloplasmin-Konzentration der Tumorpatientinnen beträgt $0,35 \mathrm{~g} / 1(\mathrm{Me}-$ dian). Mit der Festphásenantigen-Lumineszenztechnik (SPALT) wurde in den gleichen Serumproben ein
Median von $0,78 \mathrm{~g} / \mathrm{l}$ mit einer Spannbreite der Werte von 0,12 bis 1,3 bestimmt. Die statistischen Daten sowie die Verteilung der Einzelwerte und die Zuordnung dieser zu Konzentrationsklassen sind in Tabelle 3 und den Abbildungen 1 und 2 dargestellt.

Ein Vergleich der nach den drei Methoden gemessenen Caeruloplasmin-Konzentrationen zeigt, daß insbesondere im SPALT-Assay der Median der Konzentration im Serum deutlich über dem entsprechenden nach der enzymatischen und der ILMA-Methode gemessenen liegt. Im Vergleich mit dem Referenzkollektiv zeigt sich, wie auch aus Abbildung 1 ersichtlich, eine deutlich erhöhte mittlere Konzentration bei den Tumorpatientinnen, deren Werte nach dem SPALTAssay gemessen wurden. Die Caeruloplasmin-Konzentrationen im Serum von 48 Patientinnen (entsprechend $75 \%$ ) liegen oberhalb der 97,5 Percentile der gesunden Blutspender. Der Unterschied der Konzentrationen ist nach dem Mann-Whitney-U-Test für nichtparametrische Stichproben signifikant $(Z=$ 7,1).

Bei den enzymatisch bestimmten CaeruloplasminKonzentrationen fanden sich keine pathologisch erhöhten Konzentrationen. Bei den mittels der ILMATechnik bestimmten Konzentrationen sind, verglichen mit den Referenzwerten für diese Methode, nur wenige pathologisch erhöht.

Zwischen der enzymatisch gemessenen und mittels SPALT-Technik bestimmten Caeruloplasmin-Konzentration besteht keine signifikante lineare Korrelation $\left(\mathrm{r}=-0,25 ; \mathrm{A}_{\mathrm{yx}}=0,88, \mathrm{~B}_{\mathrm{yx}}=-0,3, \mathrm{~A}_{\mathrm{xy}}=0,6\right.$, $\left.B_{x y}=-0,21\right)$. Ebensowenig besteht eine Korrelation zwischen der enzymatisch und der immunoluminometrischen nach dem ILMA-Prinzip gemessenen Konzentration $\left(r=0,28 ; A_{y x}=0,23, B_{y x}=0,08, A_{x y}=\right.$ $\left.0,18, B_{x y}=0,99\right)$.

Tab. 3. Caeruloplașmin-CEA-T'TA.

Konzentrationen im Serum von Patientinnen mit Ovarial-, Collum- und Corpuskarzinom. Bestimmung des Caeruloplasmins nach drei unterschiedlichen Verfahren.

\begin{tabular}{|c|c|c|c|c|c|}
\hline - & Ënzym • & $\begin{array}{l}\text { Caeruloplasr } \\
\qquad(\mathrm{g} / \mathrm{l}) \\
\text { SPALT }\end{array}$ & ILMA & $\begin{array}{l}\text { TPA } \\
(\mathrm{U} / \mathrm{l})\end{array}$ & $\begin{array}{l}\text { CEA } \\
(\mu \mathrm{g} / \mathrm{l})\end{array}$ \\
\hline Anzahl & 64 & 64 & 64 & 64 & 64 \\
\hline Mittelwert & 0,27 & 0,74 & 0,44 & 204 & 9,2 \\
\hline $\begin{array}{l}\text { Percentile } \\
2,5 \\
16 \\
50 \text { (Median) } \\
84 \\
97,5\end{array}$ & $\begin{array}{l}0,001 \\
0,21 \\
0,27 \\
0,33 \\
0,37\end{array}$ & $\begin{array}{l}0,12 \\
0,37 \\
0,78 \\
1,06 \\
1,20\end{array}$ & $\begin{array}{l}0,12 \\
0,23 \\
0,35 \\
0,61 \\
1,20\end{array}$ & $\begin{array}{r}12 \\
55 \\
99 \\
184 \\
1596\end{array}$ & $\begin{array}{l}1 \\
1 \\
1,8 \\
16,2 \\
60\end{array}$ \\
\hline Mittelwert/Median & 0,98 & $0 ; 95$ & 1,26 & 2,06 & 5,0 \\
\hline Spannbreite & $0-0,41$ & $0,12-1,3$ & $0,12-1,47$ & $12-3000$ & $1-65$ \\
\hline
\end{tabular}




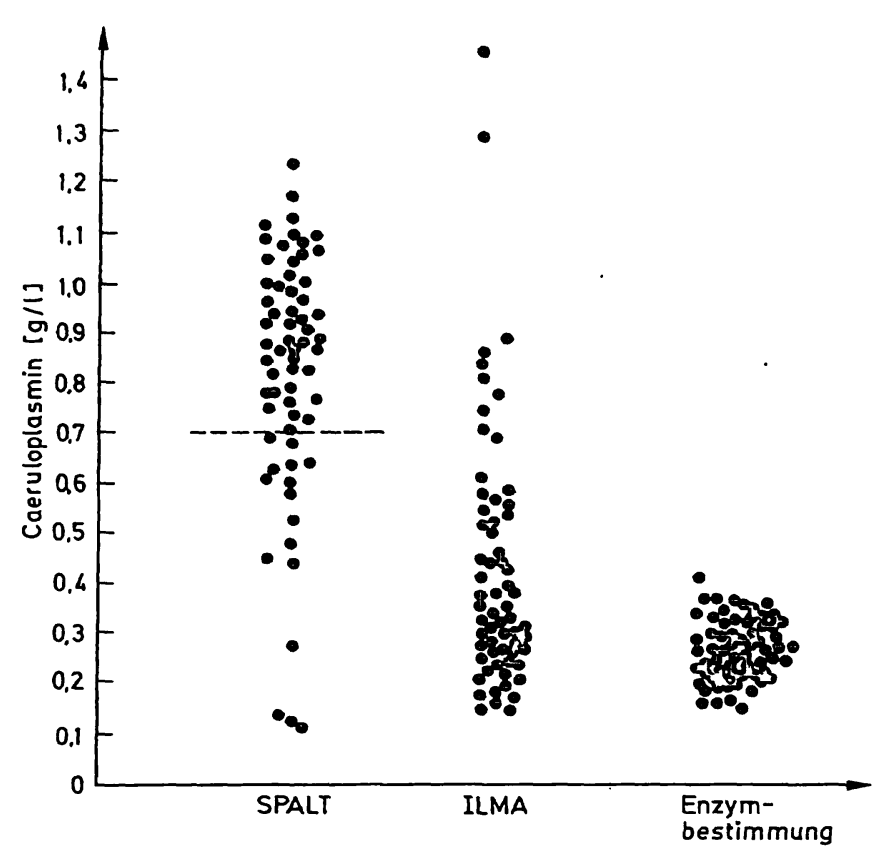

Abb. 1. Caeruloplasmin-Konzentration im Serum von Patientinnen mit Ovarial-, Collum- und Corpuskarzinom. Darstellung der nach unterschiedlichen MeBmethoden ermittelten Einzelwerte. $(---=$ Median).

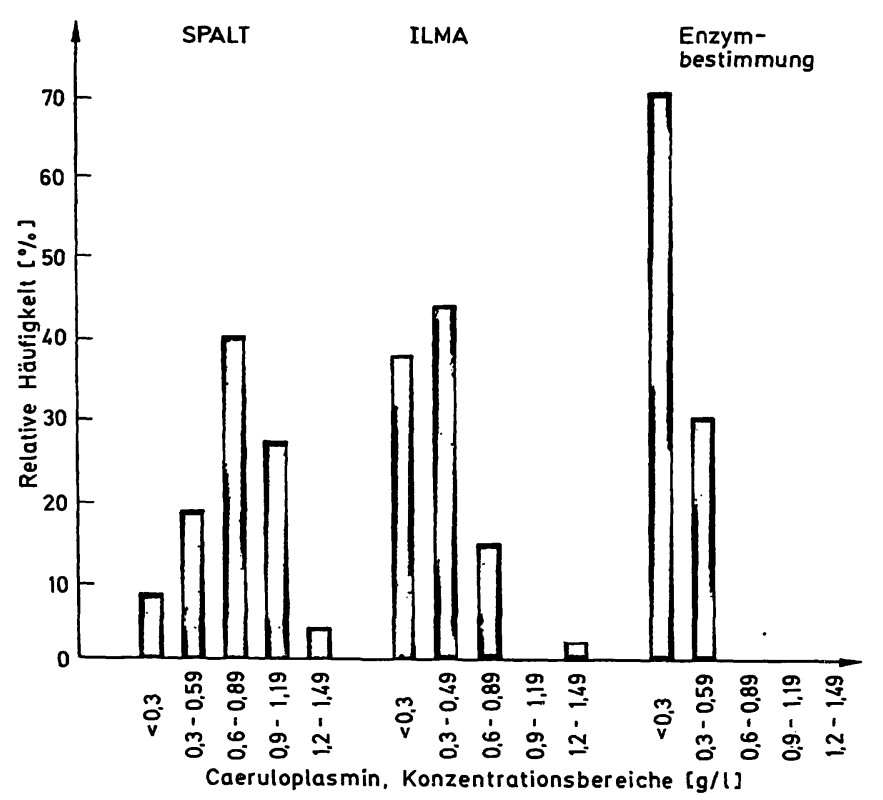

Abb. 2. Histogramme der Caeruloplasmin-Konzentrationen (siehe Abb. 1).

In Abbildung 5 ist das Elutionsprofil der Gelfiltrations-Chromatographie dargestellt. Es zeigt sich, daß mit den SPALT-Assays (SPALT 1 und 2) noch eine hohe Caeruloplasmin-Konzentration in Fraktionen nachgewiesen wird, in denen die nach dem ILMA gemessene an der unteren Nachweisgrenze oder unterhalb dieser liegt.
Der Median der gemessenen TPA-Konzentration beträgt $99 \mathrm{U} / \mathrm{l}$, der Median der CEA-Konzentration 1,8 $\mu \mathrm{g} / \mathrm{l}$ (Tab. 2). Die entsprechenden Konzentrationen von 108 Blutspendern als Referenzwerte sind in Tabelle 1 aufgeführt.

Ein Vergleich der TPA-Konzentrátionen der Karzinompatientinnen mit dem Referenzkollektiv zeigt einen erhöhten Medianwert. Dieser Unterschied ist im Mann-Whitney-U-Test für nichtparametrische Verteilung signifikant $(Z=3,65, p<0,01)$. Von den 64 Probandinnen hatten 51 TPA-Werte oberhalb des oberen Grenzwertes von $60 \mathrm{U} / 1$. Die mittlere CEAKonzentration unterscheidet sich nicht vom Vergleichskollektiv. Von 64 Patientinnen hatten lediglich 14 CEA-Werte oberhalb der 97,5 Percentile des gesunden Referenzkollektivs.

Vergleicht man die CEA- und TPA-Konzentrationen mit den entsprechenden nach verschiedenen Methoden gemessenen Caeruloplasmin-Konzentrationen, findet sich keine signifikante Korrelation.

\section{Diskussion}

Als auffälligster Befund fand sich ein signifikanter Unterschied der Konzentration von Caeruloplasmin, bestimmt nach der SPALT-Methode im Gegensatz zum ILMA-Assay und zur enzymatischen Meßmethode. Es muß angenommen werden, $\mathrm{da} ß$ mit dem SPALT auch paraneoplstisch gebildetes, Caeruloplasmin-ähnliches Protein erfaßt; wird, möglicherweise auch isolierte Proteinketten oder metabolische Abbauprodukte. Beim SPALT (Abb. 3) ist nur eine Antigen-Determinante zur Bestimmung und somit für den Ablauf des Assays notwendig, beim ILMA (Abb. 4) dagegen mindestens zwei. Somit ist die Wahrscheinlichkeit, paraneoplastisch gebildetes Eiweiß mit einer dem Caeruloplasmin identischen immunreaktiven Sequenz (z. B. isolierte Ketten) zu erfassen, größer als beim ILMA, bei dem zwei aktive unterschiedliche Zentren zur quantitativen Erfassung notwendig sind. Dies wird in den Abbildungen 3 und 4 verdeutlicht.

Das Konzentrationsprofil der Gelfiltrations-Chromatographie (Abb. 5) bestätigt, daß der SPALT eine höhere Konzentration Caeruloplasmin-identischer Aktivität erfaßt als der ILMA.

Im enzymatischen Test wird das physiologische, enzymatisch aktive Caeruloplasmin mittels Oxidase-Aktivität bestimmt. Das im SPALT bestimmte Protein mit einer dem Caeruloplasmin entsprechenden aktiven Teilsequenz zeigt in unserem Falle keine meßbar erhöhte enzymatische Aktivität. :' 
1.

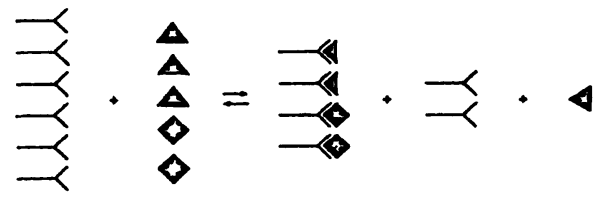<smiles>[Z]C(C)=CC(C)C(C)=O</smiles><smiles>CC1=C(C)C(=C=C=C2CCC(CCCC(C)C(C)C)CC2)CC1</smiles>

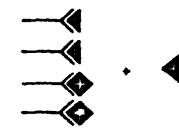

3.

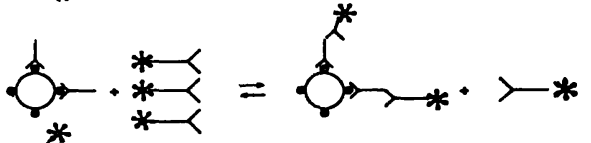

4.

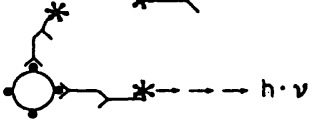

Abb. 3. Darstellung des SPALT für Caeruloplasmin ( $\diamond) \mathrm{mit}$ vermuteter Erfassung paraneoplastisch gebildeten Proteins mit Caeruloplasminantigeneigenschaft (4)

1.

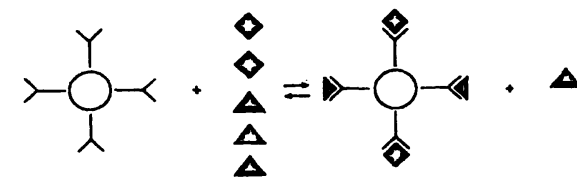

2.

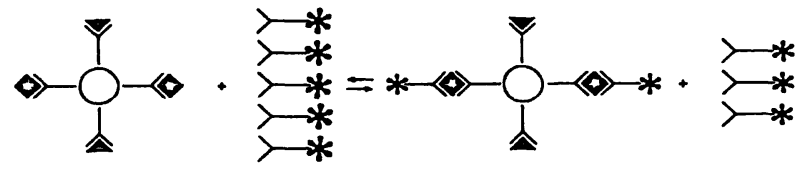

3.

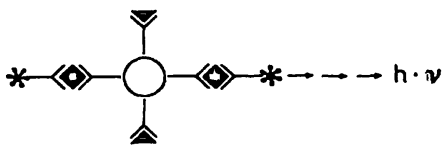

Abb. 4. Darstellung der ILMA-Meßmethode für Caeruloplasmin $(\diamond)$ mit vermuteter Erfassung paraneoplastisch gebildeten Proteins mit Caeruloplasminantigeneigenschaft (4).

Für das Ovarial- und Collum- bzw. Corpuskarzinom hat somit das im SPALT-Assay bestimmte Caeruloplasmin (bzw. das paraneoplastische Prọtein mit Caeruloplasmin identischer, immunologisch erfaßbarer Teilsequenz) Eigenschaften eines Tumormarkers.

Linder et al. (5) fanden bei Lungentumoren eine signifikante Erhöhung sowohl des enzymatisch als auch des als Antigen (Radio-Immuno-Diffusion-Methode) bestimmten Caeruloplasmins in Abhängigkeit vom Tumorausbreitungsgrad. Auch das Kupfer im Serum war deutlich erhöht. Bei der Untersuchung von $\mathrm{Pa}-$ tienten mit Brustkrebs fanden sich normale Antigenkonzentrationen, dagegen ein im Mittel erhöhter Kupfergehalt.

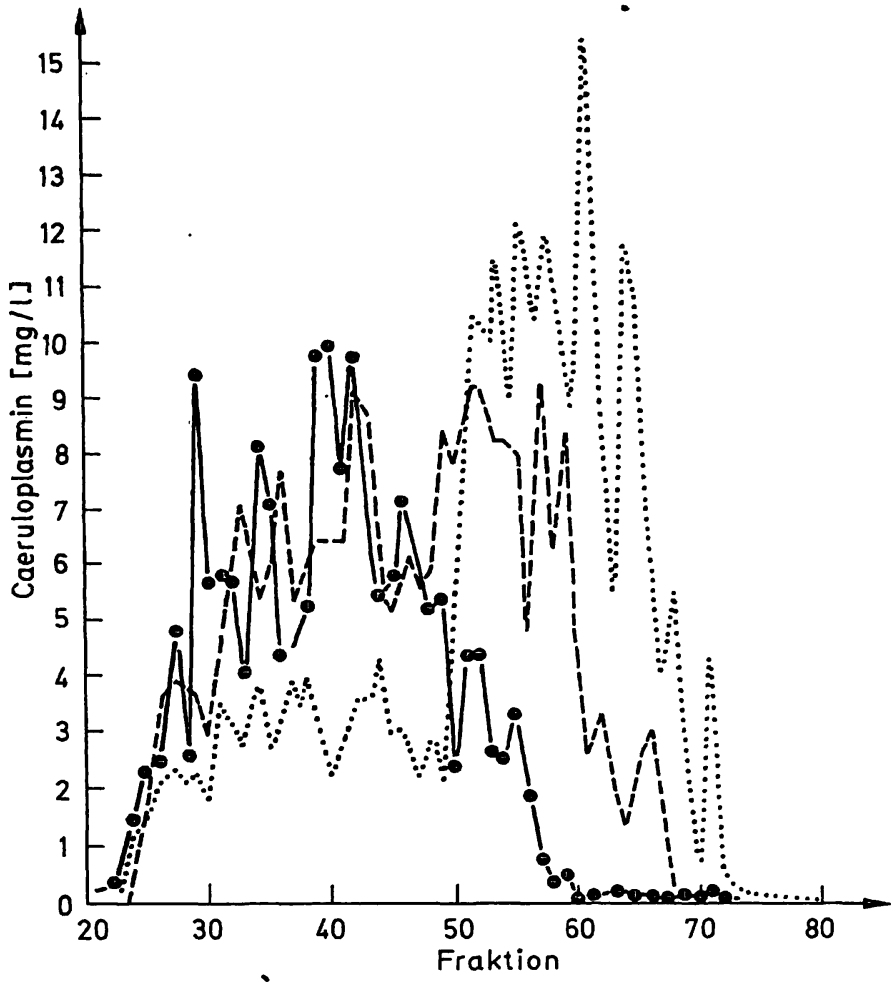

Abb. 5. Elutionsprofil einer Gelfiltrations-Chromatographie: Bestimmungen der Caeruloplasmin-Konzentration nach drei unterschiedlichen Methoden (s. Text).

$\begin{array}{ll}\ldots & \text { ILMA } \\ \ldots & \text { SPALT } 1 \\ \ldots & \text { SPALT } 2\end{array}$
Über ähnliche Ergebnisse berichteten auch andere Autoren $(1,6)$. Auch bei Patienten mit chronisch obstruktiver Lungenerkrankung können erhöhte Caeruloplasmin-Konzentrationen sowohl im immunologischen als auch im enzymatischen Assay nachgewiesen werden (5). Beim Caeruloplasmin handelt es sich somit nicht um einen tumorspezifischen Marker, bei einer Reihe anderer Erkrankungen (13) fand man parallel zur Kupferkonzentration erhöhte Konzentrationen im Serum. Am häufigsten sind Caeruloplasmin-Änderungen bei Wilson'scher Erkrankung, Niereninsuffizienz (9), verschiedenen Lebererkrankungen (8) sowie nach Einnahme von Ovulationshemmern (2) nachzuweisen. Gray \& Walker (4) fanden einen Anstieg des Caeruloplasmins beim Rezidiv eines Colon- und Sigma-Karzinoms in guter Korrelation zum CEA.

Die in unseren Studien bestimmten, im Vergleich zum Normalkollektiv erhöhten CEA- und TPA-Konzentrationen sind in Übereinstimmung mit früheren Ergebnissen (10). 


\section{Literatur}

1. Andrews, G. S. (1979) J. Clin. Pathol. 32, 325-333.

2. Briggs, M., Austin, J. \& Staniford, M. (1970) Nature 81, 225.

3. Colombo, J. P. \& Richterich, R. (1964) Schweiz. Med. Wochenschr. 94, 715-720.

4. Gray, B. N. \& Walker, C. (1983) Surgery, Gynecology and Obstretics 156, 777-780.

5. Linder, M. C., Moor, J. R. \& Wright, K. (1981) J. Natl. Cancer Inst. 67, 263-275.

6. Mailer, C., Schwatz, H. M., Konieczny, M., Ambegaonkar, S. \& Moore, V. L. (1974) Cancer Res. 34, 637-642.

7. McCombs, M. L. \& Bownsan, B. H. (1976) Biochim. Biophys. Acta 434, 452-461.
8. Ritland, S., Eiliv, S. \& Sverre, S. (1977) Scand. J. Gastroenterol. $12,81-88$.

9. Schmidt, P., Zażgornik, J., Kopsa, H. \& Kotzarek, R. (1973) Klin. Wochenschr. 51, 999-1004.

10. Wood, W. G.; Bellinger, H., Schultz, E. \& Otte, M. (1984) Ärztl. Lab. 3C, 105-114. is

11. Wood, W. G., Fricke, H., Haritz, J., Gadow, A., Krausz, H.-S., Tode, B., Strasburger, C. J. \& Scriba, P. C. (1984) J. Clin. Chem. Clin. Biochem. 22, 349-365.

12. Wood, W. G., Keller, C., Wießmann, K.-J., Krausz, H.-S., Haritz, J. \& Hantke, U. (1984) Ärztl. Lab. 30, 336-342.

13. Zlatkov, N. B., Bozlikov, B. \& Grenov, D. (1973) Arch. Dermatol. Forsch. 247, 289-294.

Dr. med. Dipl.-Chem. Thomas Schultek Klinik für Innere Medizin

Medizinische Universität zu Lübeck

Ratzeburger Allee 160

D-2400 Lübeck 1 